\title{
RECONCILING OPERATIONAL AND FINANCIAL PLANNING VIEWS IN A CUSTOMER-FUNDED ORGANIZATION: MAKING CUSTOMER- FUNDING WORK FOR NC3A
}

\author{
Jean-René COUTURE
}

\begin{abstract}
As it started its journey as a customer-funded organization, the NATO Consultation, Command and Control Agency (NC3A) needed to adopt operational and financial planning practices normally found in the commercial world. Customizing and implementing such an approach in an international governmental organization presented several challenges to those who were called to make it work. This paper provides the background, defines the problem, presents the approach to reconciling requirements and constraints in a customer-funding regime, and analyses the experience in the first years of its implementation.
\end{abstract}

Keywords: NATO, NC3A, customer-funding.

\section{Background}

I joined the NATO Consultation, Command and Control (C3) Agency (NC3A ${ }^{1}$ ) in 2002, a few short years after it was established as a customer-funded organization. It was a new NATO body resulting from the merger of two existing (budget-funded) agencies: the NATO Communications and Information Systems Agency (NACISA) and the SHAPE Technical Centre (STC). NC3A was put under a "complete" customer-funding arrangement: the Agency did not receive any budget to pay for its operation; all of its expenditures had to be covered by its revenue. In those early days of customer-funding in NATO everything had to be done: by and large, the necessary governance and management framework was still to be established.

The two main planning documents required from the Agency by its board of governors, the then NATO Consultation, Command and Control Board $\left(\mathrm{NC}_{3} \mathrm{~B}^{2}\right)$, on an annual basis, were the Statement of Planned Income and Expenditure (SPIE) and the Consolidated Programme of Work (CPOW). The former was the Agency's financial plan whilst the latter was indeed the consolidation of the programmes of work of the 
Agency's customers. ${ }^{3}$ The nomenclature used for these two essential documents reflected the "budget-funded" influence and mentality that were still very prevalent in the Agency and NATO HQ's corridors at that time. In effect, the Agency had yet to turn the corner and operate as a full fledge customer-funded organization; it would take some more years to get there.

I worked at NC3A from 2002 to 2011 in different capacity, but throughout my stay in the Agency I was involved (and eventually responsible) for the development of the operational view and its reconciliation with the financial view. My last plan for execution in 2012, and produced in the third quarter of 2011, required 444 Full Time Equivalent persons to execute and expected to generate a revenue of 93.9M€. I will refer to this 2012 Plan to illustrate my discussion. For further background, it should be noted that NC3A's mission was to "enable NATO's success through the unbiased provision of comprehensive C4ISR ${ }^{4}$ capabilities."

The aim of this paper is to discuss how the operational and financial views of the NC3A were reconciled to strengthen the governance and management of the Agency.

\section{The Problem}

In 2002, NC3A's operational and financial views were not really reconciled. The development of the CPOW and SPIE were two parallel exercises with minimum interaction between them. From a governance point of view each document was approved by a distinct committee. The "statement of planned income" of the SPIE was a high level macro view of the budgets available from the Agency's main customers; it was not generated from the operational view (the CPOW). In fact the notion of an Agency's order book ${ }^{5}$ did not really exist. Furthermore, these two planning documents were static in time and had to be re-done essentially from scratch for the following years, even though a fair percentage of the Agency's work was indeed multiyear's commitment.

Another problem was that all the work considered for revenue calculation was taken at face value and as a certainty, with no consideration of risk or likelihood of some of the work to materialize or not; to be contracted or not; or of the customer to receive its budget or not. Like in a budget-funded organization the revenue was very much considered binary: it is part of the revenue or it is not.

Finally, neither the CPOW nor the SPIE was translated into indispensable Agency's internal subordinate plans like a recruitment plan nor an order nook as I already indicated above. At that time, NC3A was basically providing consultancy services for the procurement of NATO's major IT systems and for scientific and engineering expertise to a broad range of customers. Determining the number of project managers, scientific experts or procurement officers needed to deliver the CPOW was essentially a 
tacit process conducted by middle level managers who had experience in the Agency. Could the Agency deliver more services to NATO was basically impossible to tell as the necessary numbers to answer this question were not explicit in any of the Agency's plans.

\section{The Approach}

In preparing for battle I have always found that plans are useless, but planning is indispensable.

Dwight D. Eisenhower

The diagram below represents in a nutshell the approach that was developed.

Customers'

Needs and

Funding
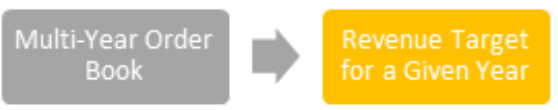

NCIA's Internal

Resources Plan

Figure 1: Approach to reconciling needs and funds.

\section{Adding up all Potential Work in an Order Book}

The first step is to collect all potential work from all possible customers. The NC3A had organized itself in a series of customer account segments to engage and work with customers helping them identified their requirements. The Agency had also adopted a comprehensive portfolio, programme and project management (P3M) approach, based on recognized methodologies and best practices. P3M significantly contributed to increase the Agency's maturity as a customer-funded organization. Putting this to use, all the work commissioned by customers was arranged in individual projects, which could be further organized together in programmes or portfolios. For example, each major customer segment's projects were organized in a customer portfolio view. In essence, the project management planning discipline allowed the Agency to more systematically and rigorously size and quantify its customers' requirements.

Two essential pieces of information were associated to each new potential project inserted into the Agency's order book: the amount of effort required to deliver the services the customers needed, planned by year; and the probability of the project to be contracted for the planning year. The amount of effort was measured in Full Time Equivalent (FTE) of person-year, which corresponded to the effort a full time employed person could deliver during one year of work. Back then, one FTE corresponded to approximately 185 to 190 person-days of effort. ${ }^{6}$ Regarding the probabil- 
ity of contracting, each project was categorized with one of the following four values and a corresponding coefficient was applied to calculate the forecasted revenue:

- Contracted: $100 \%$ of these projects were considered for the revenue calculation;

- High probability of being contracted: $90 \%$ of these projects were considered for the revenue calculation;

- Medium probability of being contracted: $50 \%$ of these projects were considered for the revenue calculation; and

- Low probability of being contracted: $30 \%$ of these projects were considered for the revenue calculation;

The chart below illustrates the NC3A potential order book as of summer $2011 \mathrm{com}$ prising 875 person-years of potential work categorized using the four categories described above. 405 person-years of work was already contracted and in the order book, and a further 470 person-years worth of work was possible with different levels of probability of contracting.

\section{Potential Muli-year Order Book}
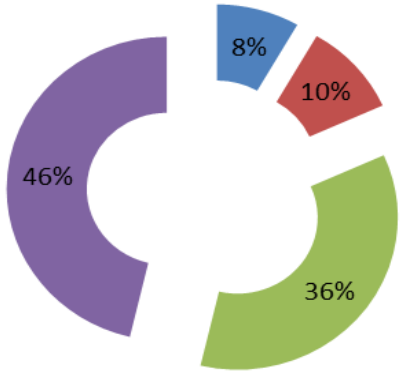

- Low probability

- Medium probability

High probability

- Contracted work

Figure 2: Example - distribution of 875 person-years of potential work.

\section{Translating Order Book in Potential Revenue}

Once the Potential Multi-Year Order Book was compiled and the Agency management was content that this order book was indeed the best possible estimate of the work expected to be commissioned to the Agency, it was time to translate FTE in a forecasted revenue expressed in Euros. 
The first step was to discount the risk from the Potential Multi-Year Order Book by applying the coefficient indicated above; for example, of the 73 person-years (FTE) of low probability work indicated in the chart above, only 22 persons-years (30\%) were considered for revenue generation.

The second step is to only consider the work that can be contracted for the planning year (2012 in the example we are considering). The final step was a management decision based on the resources immediately available in the Agency and how many more could be either recruited or contracted in time to deliver during the planning year and effectively contribute to the revenue generation of that year. In a customerfunded organization revenue is only generated by the work executed by the staff; the required amount of FTE resources need to be on board in order to start executing. This last step is indeed a management decision based on experience rather than the result of a precise calculation. As part of the customer-funding arrangement, the Agency did not have a fix personnel establishment; it could grow or shrink, within limit, to accommodate the customers' demand.

The chart on the next page illustrates the steps taken to translate the Potential MultiYear Order Book into a planning year (2012 in this case) revenue.

At this stage it was very important that the operational side of the Agency and the financial team work closely together to complete the planning effort. The forecasted revenue based on a 444 FTE effort needed to be translated in Euro based on a charge

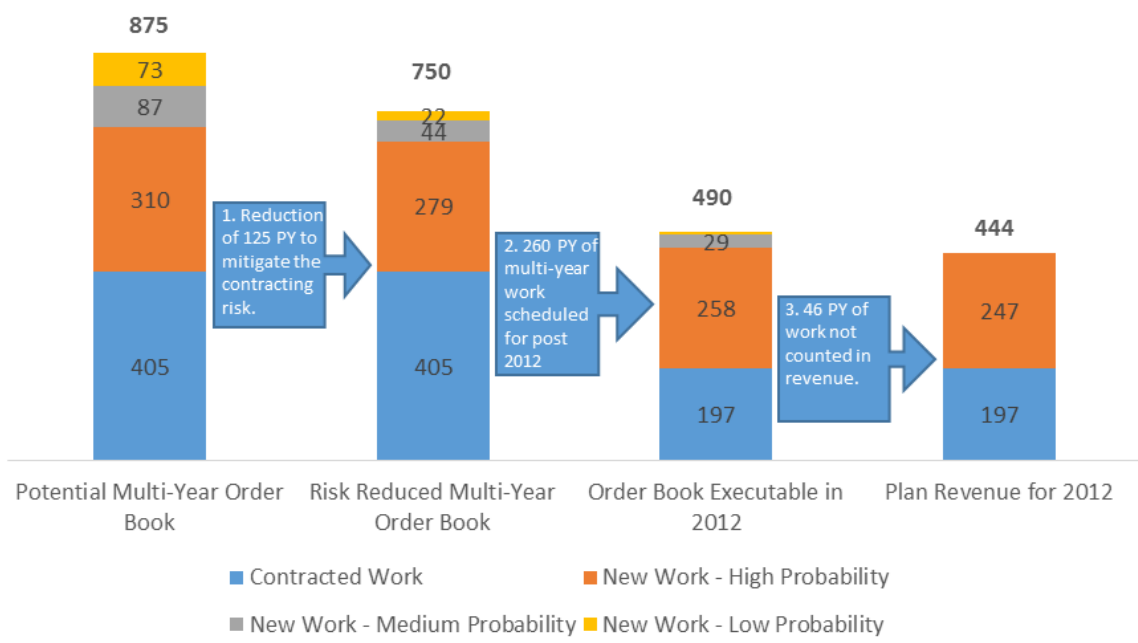

Figure 3: Illustration of the risk mitigation approach with the Order Book. 
out rate for the different grade of staff the Agency was selling. These charge out rates would obviously need to be approved by the Board of Governors.

\section{Some other considerations}

Once the above target was agreed by management, the expenditures plan could be designed with the aim to cover all of the Agency's direct and indirect costs necessary to pay for the resources delivering the work commissioned by its customer. One of the challenges was that the Agency aimed at breaking even, in order words presenting a bottom line result of $0 €$. Any surpluses or losses were more or less similarly frown upon by the governors and also the customers.

During the execution year, the achievement of the required FTEs to reach the revenue target were monitored constantly and regularly reported to management. The Agency had a time accounting system where all the work carried to deliver the services requested by the customers was recorded and could then be compiled into management reports indicating progress against the forecasted revenue. The revenue plan was further broken in monthly period in order to set internal monthly targets.

\section{Conclusion}

By adopting a customer-funded regime, the governors wanted the Agency to adopt business' best practices, increase effectiveness and efficiency, whilst being transparent to governors and customers. It took several years to evolve and mature the approach described in this paper. There was no customer-funded culture in the Agency nor with the governing committees; amongst other things, financial regulations had to be adapted to reflect the reality of customer funded. Such changes are always challenging in any organization. In a budget organization only the expenditures have to be managed during execution; the revenue (the budget) is fixed at the beginning of the year. In a customer-funded organization both revenue and expenditures have to be managed throughout the execution period. That said, I personally witnessed an Agency that was getting better and better over time in going through the planning-execution-reporting cycle.

Regular and transparent engagement at the management and governance levels was essential. Both management and governors had to become familiar and build trust in the "business numbers" presented and in the processes to arrive at them. This is particularly important in an environment such as NATO where there is a frequent rotation of high level managers and governors and where NC3A was the only organization operating in such a customer-funded regime. To facilitate trust the management assumptions guiding the planning season were eventually presented to and approved by the governors. 


\section{Notes}

1 In 2012, NC3A was merged with four organizations to form the NATO Communications and Information Agency (NCIA).

2 The NC3B is now the C3B.

3 During NC3A's 15 year history, there was an on-going debate about how the organizations commissioning work to the Agency should be called: customer, sponsor or some other name. There was a belief that using the term "customer" pigeon-holed the Agency as an outside commercial business rather than the inside-the-fence trusted partner the Agency was aspiring to be. Notwithstanding this debate the word customer is used in this paper.

4 C4ISR: Consultation, Command, Control, Communications, Intelligence, Surveillance and Reconnaissance.

5 Wikipedia defines order book as " a business's list of open, unshipped, customer orders, normally time-phased and valued at actual individual order prices that may include margin and profitability analysis."

6 One FTE discounted the number of weekend days, holidays, leave days, sick days and training days using statistical evidence from the Agency's time accounting system.

\section{About the author}

Jean-René COUTURE is a member of NATO International Staff, currently the Head of the Information Services Branch of the NATO Headquarters C3 Staff in Brussels. Prior to NATO HQ, COUTURE was the operational planner at the NC3A contributing to the development of the customer-funding practices discussed in this paper. COUTURE also served 20 years in the Canadian Army. He holds a Master in Business Administration from HEC Montréal, Canada. 